\title{
Asymptomatic postmenopausal women with sonographically thickened endometrium. What do we do?
}

\author{
Sahathevan Sathiyathasan", K. Jeyanthan, C. L. Khoo
}

Ealing Hospital, Middlesex, UK

Email: ${ }^{*}$ minisathya@hotmail.com

Received 24 August 2013; revised 22 September 2013; accepted 30 September 2013

Copyright (C) 2013 Sahathevan Sathiyathasan et al. This is an open access article distributed under the Creative Commons Attribution License, which permits unrestricted use, distribution, and reproduction in any medium, provided the original work is properly cited.

\begin{abstract}
Background: A thickened endometrium in the postmenopausal patient is a common reason for referral to gynaecology rapid access clinics under the cancer targets wait. This can often be an incidental finding in the asymptomatic patient. Investigation of this can be invasive, uncomfortable and carries significant surgical risk. Aims: This paper aims to summarise all the recent literature to see if there is any clear consensus on who should be further investigated in this select group of patients. Methods: Systematic literature review. Results: The studies varied greatly on the need for further investigation in asymptomatic women with endometrial thickness greater than $11 \mathrm{~mm}$, with some deciding on careful case selection for further investigation, and others investigating every patient. The treatment of asymptomatic patients with endometrium classified as thickened, yet under $11 \mathrm{~mm}$ and no symptoms again varied in case selection and further investigation. Endometrial polyps were mentioned in 1 paper, suggesting that asymptomatic polyps need not be removed, which is contrary to current clinical practice. Conclusion: There is no overall opinion as to the exact treatment of women with thickened endometrium. Women should be carefully triaged and all other factors taken into account before further investigation are instigated.
\end{abstract}

Keywords: Endometrial Thickness; Ultrasound; Endometrial Pathology

\section{BACKGROUND}

Endometrial carcinoma is the most common genital cancer in women. The most common presentation is with post menopausal vaginal bleeding. However, in $10 \%-$ $20 \%$ of patients this characteristic symptom is absent.

"Corresponding author.
Measurement of endometrial thickness is an important tool in the assessment of women with postmenopausal bleeding. Endometrial thickness is measured by transvaginal ultrasonography (TVUS) and thickening indicates an increased risk of hyperplasia or malignancy [1]. The role and significance of endometrial thickness measurement by ultrasound in asymptomatic women are unclear.

\section{AIMS}

To perform a detailed literature review and analysis of papers and published studies to assess what is the best management plan for asymptomatic women with endometrial thickening found on TVUS.

\section{METHODS}

A detailed search was performed using the search terms asymtamatic postmenopausal women, endometrial thickness, transvaginal ultrasound scan and endometrial thickness on PubMed, Cochrane data base were searched for relevant randomised control trial, systematic reviews and Meta analysis. These papers were analysed with regard to endometrial thickness in asymptomatic post menopausal women, interventions and outcomes, focussing on whether TVUS could be used as an adequate screening tool for detection of endometrial carcinoma.

\section{FINDINGS}

Transvaginal ultrasonography is routinely performed as part of a pelvic sonogram in postmenopausal women, and images of the endometrium, including a measurement of the endometrial thickness (ET) are frequently obtained. A thickened endometrium (usually $>4 \mathrm{~mm}$ ) is seen as an indication to proceed to further, more invasive investigations. In women without vaginal bleeding, the threshold separating normal from abnormally thickened endometrium is not known. 
In one paper, the recommendation is that in postmenopausal woman without vaginal bleeding, if the endometrium measures $>11 \mathrm{~mm}$ a biopsy should be considered as the risk of cancer is $6.7 \%$, whereas if the endometrium measures $11 \mathrm{~mm}$ or less a biopsy is not needed as the risk of cancer is extremely low. This explains the arbitrary cut off for endometrial thickness needing further endometrial investigations in this group of women.

A recent systematic review and Meta analysis with 11,000 women does not justify the use of endometrial thickness as a screening test for endometrial carcinoma and a typical endometrial hyperplasia in asymptomatic postmenopausal women not using hormone replacement therapy (HRT) [2,3].

In contrast, a Greek study comparing a retrospective analysis from January 2000 and December 2008 looked at transvaginally defined endometrial thickness (ranged between 5 and $20 \mathrm{~mm}$ ) with hysteroscopy and histopathology findings in asymptomatic postmenopausal women. This recommended hysteroscopy to follow gynaecological TVS when a thickened (ranged between 5 and $20 \mathrm{~mm}$ ) endometrium is found in asymptomatic postmenopausal women for better diagnostic and, at a later stage, therapeutic efficacy.

This view is supported by a retrospectively reviewed study, in which 850 postmenopausal women were studies and underwent hysteroscopy, focusing on 148 cases with an ultrasound indication of endometrial thickening. The findings show that the use of TVUS as a screening tool for endometrial pathology in asymptomatic postmenopausal women generates $93.2 \%$ false positive results, so that most of these women undergo this second level invasive procedure unnecessarily. The data suggests that, in asymptomatic postmenopausal women, endometrial ultrasound evaluation is not worthwhile as a screening tool, such as it is considered in common clinical practice [4].

In this respect the practice varies in different part of the world. The Society of Obstetricians and Gynaecologists of Canada recommend that woman with endometrial thickening over $11 \mathrm{~mm}$ and other positive findings on ultrasound, such as increased vascularity, inhomogeneity of endometrium or particulate fluid should be referred to a gynaecologist for further investigations.

Further to this, decisions about further investigations should be made on a case-by-case basis in asymptomatic women with increased endometrial thickening and risk factors for endometrial cancer such as obesity, hypertension, and late menopause. This recommendation additionally stats that asymptomatic women on tamoxifen should not have TVUS assessment of endometrial thickness.

It further goes on to conclude that should endometrial polyps be noted on TVUS, surgical removal is not always required. Should a woman be found to have asymptomatic polyps, decision for intervention should be according to size of the polyp, age, and other risk factors.

The 2011 Map of Medicine pathway for endometrial cancer 2011 suggests that in asymptomatic postmenopausal women who are not receiving hormone therapy an endometrial thickness between $5 \mathrm{~mm}$ and $11 \mathrm{~mm}$ requires further investigations based on clinical opinion regarding risk of underlying pathology. However, an endometrial thickness above $11 \mathrm{~mm}$ warrants further investigation with hysteroscopy/biopsy.

Finally there was a prospective study looking at 1500 consecutive asymptomatic postmenopausal women receiving TVUS assessment. 92\% of asymptomatic postmenopausal women not on hormone replacement therapy had an ET of $\leq 5 \mathrm{~mm}$. The mean ET was $3.71 \pm 1.9 \mathrm{~mm}$. However, a significant group, $6.7 \%$, had an endometrial lining suspicious for polyp. These women had a significant increase in mean age and ET [5].

\section{CONCLUSIONS}

Asymptomatic endometrial thickening found on ultrasound examination in postmenopausal women often creates a clinical management dilemma.

Women with risk factors for endometrial cancer and endometrial thickening, such as tamoxifen use, obesity, hypertension and late menopause should be triaged and investigated on an individual basis. Endometrial Polyps found in asymptomatic postmenopausal women need not be removed routinely. However, factors such as polyp size and histopathology of endometrium and patient age must be considered in the decision for polypectomy.

There should be a justification to investigate patients with no symptoms but thickened endometrium found on TVUS. The investigations are not risk free, and serious complications such as bowel injury and uterine perforation have been reported in the literature. Therefore, carefulness and consideration may reduce anxiety, and risk of procedural complication to the postmenopausal patient.

\section{REFERENCES}

[1] Schmidt, T., Breidenbach, M., Nawroth, F., Mallmann, P., Beyer, I.M., Fleisch, M.C. and Rein, D.T. (2009) Hysteroscopy for asymptomatic postmenopausal women with sonographically thickened endometrium. Maturitas, 62, 176-178.

[2] Curcić, A., Durdević, S., Mladenović-Segedi, L., Grujić, Z. and Visnjevac, N. (2009) Ultrasound in screening of endometrial carcinoma in asymptomatic postmenopausal women. Medicinski Pregled, 62, 263-267.

[3] SOGC Clinical Practice Guideline, No. 249, 2010.

[4] Gambacciani, M., Monteleone, P., Ciaponi, M., Sacco, A. 
and Genazzani, A.R. (2004) Clinical usefulness of endometrial screening by ultrasound in asymptomatic postmenopausal women. Maturitas, 48, 421-424.

[5] Hartman, A., Wolfman, W., Nayot, D. and Hartman, M.
(2013) Endometrial thickness in 1500 asymptomatic postmenopausal women not on hormone replacement therapy. Gynecologic and Obstetric Investigation, 75, 191-195. http://dx.doi.org/10.1159/000347064 